\title{
Clinical and epidemiological features of acute follicular conjunctivitis with special reference to that caused by herpes simplex virus type 1
}

Eiichi Uchio, Satoshi Takeuchi, Norihiko Itoh, Noriko Matsuura, Shigeaki Ohno, Koki Aoki

\begin{abstract}
Backgroundlaims-It is reported by the national surveillance of ocular infectious diseases in Japan that $4.3 \%$ of cases of epidemic keratoconjunctivitis (EKC) diagnosed clinically were caused by herpes simplex virus (HSV). Clinical and virological studies of patients with HSV conjunctivitis were carried out.

Methods-The study population consisted of 478 patients with acute follicular conjunctivitis. Virological analysis was carried out for adenovirus (Ad) and HSV by the cell culture method and fluorescein antibody (FA) method. Polymerase chain reaction for Chlamydia trachomatis was also carried out.

Results-From 23 patients, HSV type 1 was isolated but Ad or C trachomatis was not isolated. $87 \%$ of cases were unilateral. Most cases showed clinical resolution within 9 days. Early corneal lesions and preauricular lymphadenopathy were less frequent in HSV conjunctivitis than in adenoviral conjunctivitis, especially that due to subgenus $D$. No case showed a positive result for HSV by the FA method using conjunctival swabs; however, the FA test was positive in all strains isolated by cell culture.

Conclusions-These results indicate that it is difficult clinically to differentiate HSV conjunctivitis from adenoviral conjunctivitis in the acute stage, since the clinical features of adenoviral conjunctivitis are similar to those of HSV conjunctivitis. A biological difference may exist between HSV strains causing keratitis and conjunctivitis.

(Br f Ophthalmol 2000;84:968-972)
\end{abstract}

Viral conjunctivitis in east Asia including Japan, Korea, and Taiwan ${ }^{1}$ is caused mainly by adenovirus (Ad), affects approximately one million people in Japan per year, ${ }^{2}$ and has gained recognition as a major international public health problem in these regions. The causative agents of acute conjunctivitis clinically diagnosed as epidemic keratoconjunctivitis (EKC) have recently been established in Japan. ${ }^{23}$ Adenoviruses are the most prevalent causative agent of viral conjunctivitis and were isolated from $91.2 \%$ of cases of clinically diagnosed EKC in which the aetiological agent was determined virologically. ${ }^{2}$ Herpes simplex virus (HSV) was isolated in $4.3 \%$ and Chlamy- dia trachomatis was isolated in $2.5 \% .^{2}$ Other viruses, including varicella zoster virus and enteroviruses, were detected in $1.3 \%{ }^{2}$

Although herpesvirus infection is frequently diagnosed in dendritic or geographic corneal ulcers, disciform keratitis, and keratouveitis the virus is very rarely implicated as a cause of conjunctivitis alone without corneal or lid lesions. There have been few papers describing the epidemiological features of HSV conjunctivitis, ${ }^{3-5}$ and its clinical characteristics are not well clarified.

In this paper, the results of clinical and epidemiological studies of acute conjunctivitis caused by HSV are presented. Specific virological characteristics of the clinical isolates determined by the fluorescein antibody (FA) method (MicroTrak) are also presented.

\section{Materials and methods}

\section{CLINICAL SPECIMENS}

The study population consisted of 478 patients (226 men and 252 women, mean age 34.6 years) whose diagnosis was acute follicular conjunctivitis, suspected to be viral in origin at an eye clinic in Sapporo, Japan, from August 1992 to November 1997. These were a consecutive series of patients. The clinical data of all patients whose specimens were included in this study were examined retrospectively to investigate the correlation between results of laboratory tests and clinical diagnosis. Recurrent lesions of ocular herpes may present as blepharitis, blepharoconjunctivitis, or conjunctivitis alone. Thus, so as not to overlook lid vesicles, the lids and lid margins were carefully examined for herpetic vesicles, and cases with herpetic blepharitis or blepharoconjunctivitis as well as typical herpetic corneal ulcers were excluded. The clinical severity of conjunctivitis was classified as mild, moderate, or severe according to the criteria defined previously. ${ }^{6}$ Specimens were scraped from the lower palpebral conjunctiva with three cotton swabs. One of the cotton swabs was placed in $4.5 \mathrm{ml}$ of transport medium for polymerase chain reaction (PCR) (10 mM TRIS ( $\mathrm{pH} 8.0), 1 \mathrm{mM}$ ethylenediamine tetra-acetic acid (EDTA)). The second and third cotton swabs were used for rapid diagnosis of Ad and HSV, and placed in Bartels chlamydial transport medium (Bartels, Bellvue, WA, USA), and each specimen was vortexed and divided into aliquots for Ad $(0.1 \mathrm{ml})$ and $\mathrm{HSV}(0.1 \mathrm{ml})$ isolation. 
DIRECT SPECIMEN IDENTIFICATION OF

ADENOVIRUS AND HERPESVIRUS

Enzyme immunoassay (EIA) (Adenoclone, Cambridge BioScience, Worcester, MA, USA) to detect Ad antigen was carried out on the conjunctival specimens according to the manufacturer's instructions. The FA method MicroTrak HSV-1/HSV-2 direct specimen identification test (Syva Co, Palo Alto, CA, USA) $)^{78}$ was used for direct immunofluorescence test to detect HSV type 1 in conjunctival scrapings.

\section{ADENOVIRUS ISOLATION}

Swabs from the conjunctiva of symptomatic eyes were inoculated onto Hep-2 cells and HEL cells, which were examined for the presence of a cytopathic effect (CPE) for up to 21 days. Infected cells were identified by immunofluorescent antibody technique with Ad monoclonal antibody (Chemicon International, Inc, Temecula, CA, USA). The isolates were identified by standard neutralisation test (NT). Ad type 1-41 antisera were obtained from the American Type Culture Collection and the Center for Disease Control (CDC).

HERPESVIRUS ISOLATION

Laboratory diagnosis of HSV infection was made by means of cell culture. Swabs from the conjunctiva were inoculated onto Vero (African green monkey kidney) cell monolayers and examined for the presence of CPE showing specific plaque morphology of HSV. The virus group of isolates was identified by microplate neutralisation test with CDC reference guinea pig antisera as described elsewhere. ${ }^{9}$ In addition, HSV type specific monoclonal antibodies conjugated with fluorescein isothiocyanate (FITC) of MicroTrak HSV-1/HSV-2 culture confirmation/typing test (Syva) were used for direct immunofluorescence test of HSV infected Vero cells. The anti-HSV-1 reagent of MicroTrak HSV-1 contains a single monoclonal antibody which recognises the glycoprotein (g) C of HSV-1. ${ }^{7}$ Vero cells infected with the HSV strains were harvested for 24-48 hours and washed twice with phosphate buffered saline (PBS). Using these strains, the FA test was carried out according to the manufacturer's instructions.

DETECTION OF CAUSATIVE AGENTS OF

NON-ADENOVIRAL AND NON-HERPESVIRAL

CONJUNCTIVITIS

The causative agent of samples that showed a negative result in isolation of Ad or HSV was analysed by PCR for Chlamydia trachomatis. To detect $C$ trachomatis, two step PCR using two pairs of primers was carried out on the DNA template from the conjunctival specimens, and the amplified PCR product was analysed by $1.2 \%$ agarose gel electrophoresis and ethidium bromide staining as described previously by our group. ${ }^{10}$

\section{Results}

AETIOLOGICAL DISTRIBUTION OF ACUTE

FOLLICULAR CONJUNCTIVITIS

Out of 478 samples, 23 samples from patients with clinical signs of EKC $(4.8 \% ; 11$ men and 12 women) were positive for HSV-1 by cell culture method. Three hundred and eighty four patients $(80.3 \%)$ were diagnosed as having adenoviral conjunctivitis by the cell culture method. The sensitivity of EIA for Ad (Adenoclone) was 53.6\% (206/384), while the specificity was $100 \%$ (94/94). In contrast, no case showed a positive result of direct specimen identification test for HSV by FA method (MicroTrak). Out of 71 non-herpesviral and non-adenoviral samples, 27 specimens $(5.6 \%)$ were identified as positive for $C$ trachomatis. Three specimens showed evidence of dual infection. From two specimens, both adenovirus and $C$ trachomatis were isolated, and one specimen showed a positive result for HSV-1 and $C$ trachomatis. These cases were included in the adenoviral and herpesviral conjunctivitis groups, respectively. No case was identified as HSV-2. In the other 44 specimens (9.2\%), no virus or chlamydia was detected. Although the causative agents in these patients with acute follicular conjunctivitis are unknown, bacteria and allergy are possible candidates.

\section{SEASONAL VARIATION}

Of these 23 patients with herpesvirus conjunctivitis, six presented in August, four in July, three in June, and two each in February, April, and October. The highest incidence from June to August was similar to that observed for adenoviral conjunctivitis in Japan.

\section{AGE DISTRIBUTION}

The age of patients with HSV conjunctivitis ranged from 1 to 71 years (mean 39.3 years). The 50-59 year age group comprised the highest $(6 / 23 ; 26 \%)$ proportion of cases among all age groups. The age distribution was similar to that of adenoviral conjunctivitis (data not shown) with $65 \%$ occurring in the 20-59 year age group and only three children aged 1, 6, and 8 years.

\section{LATERALITY, TREATMENT, AND DURATION OF} CONJUNCTIVITIS

Eighty seven per cent (20/23) of cases were unilateral. Nine eyes $(34.6 \%)$ were treated with topical steroids and these cases showed serious clinical findings, such as superficial punctate keratitis or diffuse subepithelial corneal opacities. Most cases $(19 / 23 ; 82.6 \%)$ were treated with topical antibiotics. The duration of illness is shown in Figure 1. In two patients, the duration of illness could not be determined, because they visited our clinic only once. Most cases $(96 \%)$ showed clinical resolution within 9 days (mean 8.1 days), with a peak on days 7 and $8(12 / 23 ; 52 \%)$. One patient, from whom $C$ trachomatis was also detected by PCR, took a longer period (32 days) for resolution.

\section{SOURCE OF INFECTION}

Of these 23 patients, four $(17.4 \%)$ had been in close contact with a friend or colleague suffer- 


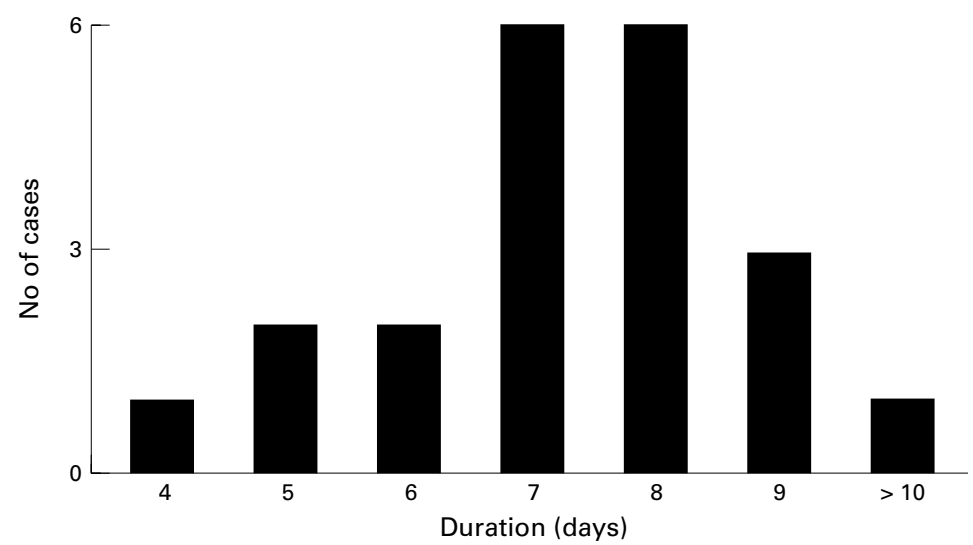

Figure 1 Duration of conjunctivitis. Most cases showed clinical resolution within 9 days (mean, 8.1 days).

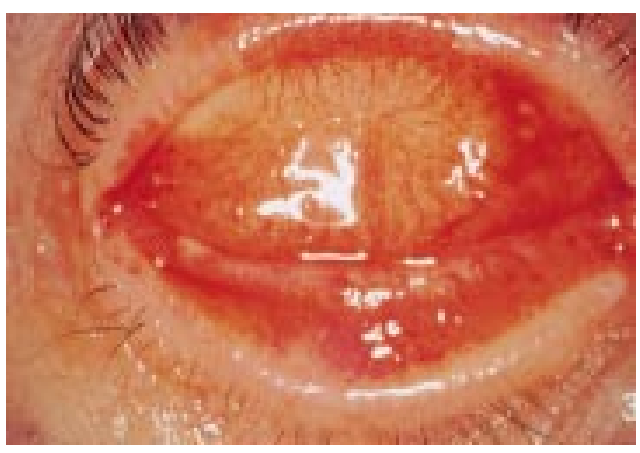

Figure 2 Moderate case of herpes simplex virus conjunctivitis. A 66 year old man, 4 days after the onset. Dot haemorrhages are seen on the palpebral conjunctiva.

ing from conjunctivitis. The second largest proportion of patients $(3 / 23 ; 13.0 \%)$ contracted the infection from a family member. The other 16 had not been aware of contact with conjunctivitis, upper respiratory infection, or urogenital disease in their family, friends, or colleagues, and had not attended a hospital or clinic or visited a swimming pool.

EXTRAOCULAR FINDINGS

Ten of the 23 patients (43.5\%) had enlarged preauricular nodes at the first visit. No glands were visibly enlarged, and no submandibular or cervical lymphadenopathy was observed. Six patients $(26.1 \%)$ had rhinitis or sore throat during the clinical course. No case had a history of herpes keratitis, and most cases had no history of blepharitis due to HSV-1 or herpes labialis.

\section{SYMPTOMS AND SIGNS}

Out of 23 cases, $19(82.6 \%)$ complained of discharge, and $12(52.2 \%)$, nine $(39.1 \%)$, and seven $(30.4 \%)$ complained of itching, sensation of foreign body, and lacrimation, respectively, in the affected eye during the early stage of the infection. Conjunctival hyperaemia was present in 23 eyes (88.5\%). Corneal involvement, such as superficial punctate keratitis, occurred in eight patients $(30.8 \%)$. None of the patients developed dendritic keratitis in the clinical course. Most cases showed mixed follicular and papillary conjunctivitis with an increasing haemorrhagic component with increasing clinical severity. Moderate conjuncti-

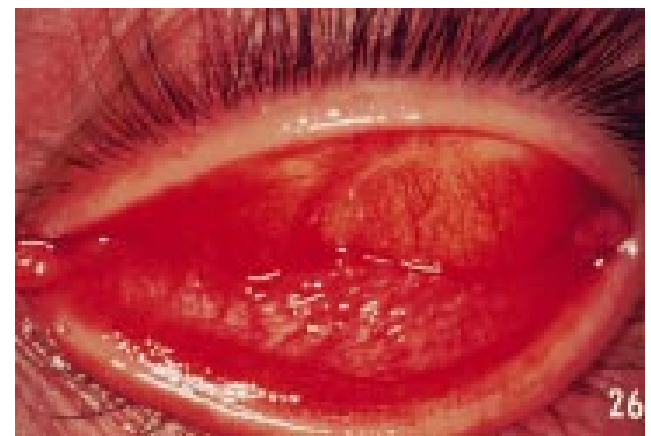

Figure 3 Moderate case of herpes simplex virus conjunctivitis. A 28 year old man, 3 days after the onset. Conjunctival follicles and hyperaemia are evident on the lower palpebral conjunctiva.

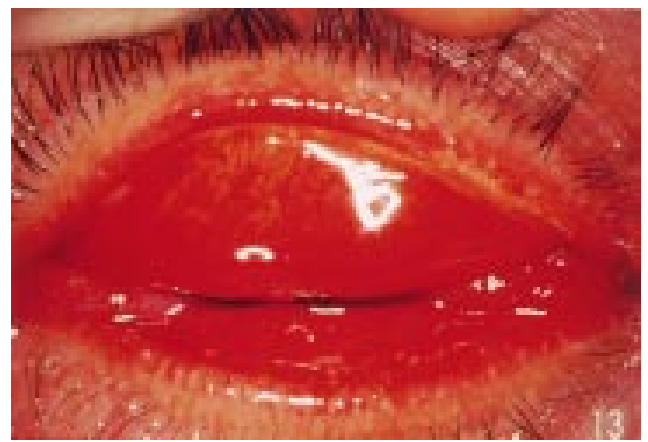

Figure 4 Severe case of herpes simplex virus conjunctivitis. A 56 year old woman, 5 days after the onset. Diffuse subconjunctival haemorrhage is present.

vitis was found in 12 eyes (46.2\%) (Figs 2 and 3 ), and severe conjunctivitis in 14 eyes $(53.8 \%)$ (Fig 4). No case of mild conjunctivitis was observed.

IMMUNOFLUORESCENCE TEST

In the immunofluorescence test using FITC labelled anti-HSV monoclonal antibodies on infected Vero cells, MicroTrak herpes antiHSV-1/HSV-2 reagents recognised the presence of HSV antigen in all clinical isolates and specific fluorescence was observed.

\section{Discussion}

It has been reported that the incidence of HSV ocular infection presenting as acute follicular conjunctivitis in the absence of corneal or lid signs in cases of clinically diagnosed EKC is $1.4-7 \% .^{2-411}$ The epidemiological frequency of our cases of HSV conjunctivitis was similar to that of previous reports.

The age of patients with HSV conjunctivitis ranged from 1 to 71 years, and three cases were under 10 years $-1,6$, and 8 years. Thus, not only cases of recurrent ocular HSV infection but also those of primary ocular infection may be included. In children and adults, primary HSV infection of the eye may often present as blepharitis with vesicles of the lid margin, often associated with conjunctivitis. ${ }^{12}$ The recurrent lesions of ocular herpes include blepharitis, blepharoconjunctivitis, and conjunctivitis alone. Since we did not measure serum antibody level to HSV, it is impossible to determine whether infection was primary or recurrent. However, it has been reported that the reliability of serological tests for the diagnosis 
of HSV infection is limited. ${ }^{13}$ Our results, therefore, suggest that there is no significant difference in the clinical features of HSV conjunctivitis between primary and recurrent ocular infections.

Compared with past reports of the clinical features of adenoviral conjunctivitis, ${ }^{3}{ }^{14-16} \mathrm{HSV}$ conjunctivitis in the present study showed similar patterns of seasonal variation, age distribution, and source of infection. The clinical features of HSV conjunctivitis-moderate follicular conjunctivitis, sometimes with preauricular node swelling and upper respiratory tract signs, and rarely with corneal involvement-are different from those of adenoviral conjunctivitis due to group D subgenera, such as types 8,19 , and 37 , which shows severe follicular conjunctivitis, sometimes with pseudomembrane and scarring of the conjunctiva, and commonly with subepithelial punctate keratitis but with little in the way of systemic disease. ${ }^{314}{ }^{15}$ However, the clinical features of adenoviral conjunctivitis due to group B subgenera, such as types 3, 7, and 11, are similar to those of HSV conjunctivitis. ${ }^{317}$ Although the low rate of bilateral illness (13.0\%) and short duration (mean 8.1 days) may help to discriminate HSV conjunctivitis from adenoviral conjunctivitis, it seems almost impossible to differentiate them clinically, especially in the early clinical stage.

There are several problems in the diagnosis of HSV conjunctivitis at the ophthalmological outpatient clinic. Firstly, uncommon cases of HSV ocular infection presenting as acute follicular conjunctivitis are clinically indistinguishable from other more likely causes, such as adenoviruses and C trachomatis. Secondly, the cells for culture isolation of HSV, such as Vero, HEK (human embryonic kidney), and PRK (primary rabbit kidney), are different from those for adenovirus, Hep-2, HeLa, and A549. Therefore, if sensitive cells are not adopted for culture isolation of $\mathrm{HSV}$ it cannot be isolated. Thirdly, a current direct specimen identification (rapid diagnostic) test for HSV, the FA kit (MicroTrak HSV-1/HSV-2), was unable to detect HSV antigen in conjunctival scrapings, although it was able to detect HSV in cell culture samples. PCR is a recently developed rapid and sensitive laboratory diagnostic method for the diagnosis of herpesvirus ${ }^{18}$; however, PCR can be conducted only in laboratories equipped with special instruments and reagents for molecular biological studies. It is reported that the sensitivity of the MicroTrak HSV-1/HSV-2 direct specimen identification test is $81.8 \%$, which is somewhat lower than that of the culture method, ${ }^{19}$ but it gives results within 2 hours after specimen collection and its staining is quite bright, indicating that it is very useful for rapid diagnosis of HSV infection, ${ }^{19}$ and MicroTrak is widely used in the diagnosis of herpetic keratitis. ${ }^{20}$

It has been reported that a $\mathrm{gC}$ negative mutant of HSV-1 was isolated from a patient with recurrent herpetic stromal keratitis. ${ }^{20-22} \mathrm{It}$ is known that $\mathrm{gC}$ is necessary for HSV attachment to cells. ${ }^{23}$ The reason why our strains did not react with MicroTrak HSV-1/HSV-2 direct specimen identification test but showed a positive result in MicroTrak culture confirmation/ typing test is unclear. However, a possible explanation is that the amount of viral antigen on conjunctival scrapings is not sufficient to give a positive result in the MicroTrak direct specimen identification test. A mutant strain of HSV-1 that lacks amino acids 33-123 at the $\mathrm{N}$-terminal end of $\mathrm{gC}$ has been reported, and it binds less well to cells than strains containing truncated forms of $\mathrm{gC}$, but exhibits normal growth properties. ${ }^{24}$ Further analysis is necessary to determine whether the clinical strains described in our study express full length $\mathrm{gC}$ or lack some of the amino acids of gC.

The mechanism by which these strains cause acute follicular conjunctivitis without inducing corneal or lid lesions is also unclear. Although $34.6 \%$ of cases were treated with topical steroids, no case developed herpetic keratitis, such as dendritic ulcer or keratouveitis. The above mentioned hypothesis that the clinical strains described in our study lack some of the amino acids of $\mathrm{gC}$ could be one explanation why these strains lack pathogenicity to cause herpetic corneal lesions even if topical steroids are given during the clinical course of conjunctivitis. Further studies including characterisation of $\mathrm{gC}$ and analysis of the mechanism of attachment of HSV to conjunctival cells are needed.

This work was supported by a grant in aid for encouragement of young scientists (09771456) from the Ministry of Education, Science, Sports and Culture of Japan. We thank Dr W Gray for Science, Sports and Culture of Japan.
the critical review of this manuscript.

1 Ishii K, Nakazono N, Fijinaga K, et al. Comparative studies on aetiology and epidemiology of viral conjunctivitis in three countries of east Asia-Japan, Taiwan and South Korea. Int f Epidemiol 1987;16:98-103.

2 Infectious Agents Surveillance Center of Japan. Viruses isolated from the eye, Japan, 1990-1994. Infectious Agents Surveillance Report 1995;16:97-8.

3 Aoki K, Kawana R, Matsumoto I, et al. Viral conjunctivitis with special reference to adenovirus type 37 and enterovirus 70 infection. Fpn f Ophthalmol 1986;30:158-64.

4 Harding SP, Mallinson H, Smith JL, et al. Adult follicular conjunctivitis and neonatal ophthalmia in a Liverpool eye conjunctivitis and neonatal ophthalmia in a

5 Belongia EA, Goodman JL, Holland EJ, et al. An outbreak of herpes gladiatorum at a high-school wrestling camp. N Engl 7 Med 1991;325:906-10.

6 American Academy of Ophthalmology. Ophthalmology basic and clinical science course. Section 7. External disease and cornea. San Francisco: American Academy of Ophthalmology, 1982:24.

7 Goldstein LC, Corey L, McDougall JK, et al. Monoclonal antibodies to herpes simplex viruses: use in antigenic typing and rapid diagnosis. F Infect Dis 1983;47:829-37.

8 Nowinski RC, Tam MR, Goldstein LC, et al. Monoclonal antibodies for diagnosis of infectious diseases in humans. Science 1983;219:637-44.

9 Tada A, Yoshino K. A new microplate neutralization test for typing of herpes simplex virus. Microbiol Immunol 1978;22: typing of
$415-26$.

10 Isobe K, Aoki K, Itoh N, et al. Serotyping of Chlamydia trachomatis from inclusion conjunctivitis by polymerase chain reaction and restriction fragment length polymorphism analysis. Ipn f Ophthalmol 1996;40:279-85.

11 Darougar S, Wishart MJ, Viswalingham ND. Epidemiological and clinical feature of primary herpes virus ocular infection. Br f Ophthalmol 1985;69:2-6.

12 Dawson CR, Togni B. Herpes simplex eye infections: clinical manifestations, pathogenesis and management. Surv Ophthalmol 1976;21:121-35.

13 Field PR, Ho DW, Irving WL, et al. The reliability of serological tests for the diagnosis of genital herpes: a critique. Pathology 1993;25:175-9.

14 Hierholzer JC, Guyer B, O'Day DM, et al. Adenovirus type 19 keratoconjunctivitis. N Engl f Med 1974;290:1436.

15 Darougar S, Quinlan MP, Gibson JA, et al. Epidemic keratoconjunctivitis and chronic papillary conjunctivitis in London due to adenovirus type 19. Br f Ophthalmol 1977; 61:76-85. 
16 Swenson PD, Lowens MS, Celum CL, et al. Adenovirus types 2,8 , and 37 associated with genital infections in patients attending a sexually transmitted disease clinic. $f$ Clin Microbiol 1995;33:2728-31.

17 Buehler JW, Finton RJ, Goodman RA, et al. Epidemic keratoconjunctivitis: report of an outbreak in an ophthalmology practice and recommendations for prevention. Infect Control 1984;5:390-4.

18 Aono T, Murakami S, Yanagihara N, et al. Detection of human alpha-herpesvirus DNA using consensus primers and specific probes. Acta Otolaryngol (Stockh) 1994; 514(suppl):132-4.

19 Kawana T, Kurata T, Sata T, et al. Detection of herpes simplex virus infection by using FITC-labeled monoclonal antibodies (MicroTrak method). Kansenshogaku Zasshi 1987;61:1030-7.

20 Kumano Y, Yamamoto M, Inomata $\mathrm{H}$, et al. Recurrent herpetic keratitis: Failure to detect herpes simplex virus infec- tion using Syva MicroTrak ${ }^{\mathrm{TM}}$ HSV-1/HSV-2 direct specimen identification/typing test. Ophthalmologica 1990;201: 169-73.

21 Hidaka Y, Sakuma S, Kumano Y, et al. Characterization of glycoprotein C-negative mutants of herpes simplex virus type 1 isolated from a patient with keratitis. Arch Virol 1990;113:195-207.

22 Sakai Y, Minagawa H, Ishibashi T, et al. Stromal keratitis induced by a unique clinical isolate of herpes simplex virus type 1. Ophthalmologica 1994;208:157-60.

23 Svennerholm B, Jeansson S, Vahlne A, et al. Involvement of glycoprotein $\mathrm{C}(\mathrm{gC})$ in adsorption of herpes simplex virus type 1 (HSV-1) to the cell. Arch Virol 1991;120:2739.

24 Tal-Singer R, Peng C, Ponce de Leon M, et al. Interaction of herpes simplex virus glycoprotein $\mathrm{gC}$ with mammalian cell surface molecules. 7 Virol 1995;69:4471-83. 